\title{
Assessment of quality indicators among nurse practitioners performing upper endoscopy
}

\section{(ㄷ)(1) $\odot$}

Authors

Jeffrey M. Baumgardner, Justin L. Sewell, Lukejohn W. Day

Institution

Division of Gastroenterology, Zuckerberg San Francisco

General Hospital and Trauma Center, San Francisco,

California, United States

submitted 7.3.2017

accepted after revision 21.6.2017

Bibliography

DOI https://doi.org/10.1055/s-0043-115384 |

Endoscopy International Open 2017; 05: E818-E824

(c) Georg Thieme Verlag KG Stuttgart · New York

ISSN 2364-3722

Corresponding author

Jeffrey M. Baumgardner, Zuckerberg San Francisco General

Hospital and Trauma Center, 1001 Potrero Ave,

San Francisco CA 94110

Fax: 1-415-206-5199

Jeffrey.baumgardner@ucsf.edu

\section{ABSTRACT}

Background and study aims Limited international data have shown that non-physicians can safely perform upper endoscopy, but no such study has been performed in the United States. Our aim was to assess the quality of outpatient upper endoscopies performed by nurse practitioners (NPs).
Patients and methods Retrospective chart review of upper endoscopies performed by 3 NPs between 2010 and 2013 was performed. Comparisons among all NPs performing upper endoscopy and assessment of individual NP performance over time with respect to quality indicators were performed.

Results Three NPs performed 333 upper endoscopies (distribution of 166,44 , and 123 , respectively). Of the cases, $98.2 \%$ s were successfully completed to the second portion of the duodenum. In most cases, photo-documentation of required anatomical landmarks was performed: GE junction (84.2\%), GE junction in retroflexed view (84.2\%), antrum (82.1\%) and duodenum (80.9\%). Photo-documentation improved with increasing experience. NPs appropriately performed biopsies for specific medical conditions: 10/11 (90.9\%) gastric ulcers were biopsied and 63/66 (95.5) of patients with iron deficiency had duodenal biopsies performed for celiac disease. A physician endoscopist was required during the procedure $22.5 \%$ of the time. Important parameters such as documenting informed consent (100\%) and documenting a discharge plan (99.4\%) in the procedure reports were overwhelming present. There was a single adverse event during the study period.

Conclusion In the first US study of NPs performing upper endoscopy, they were able to perform high-quality and safe upper endoscopies. These findings support incorporation of non-physicians alongside physicians to help meet the growing demand for endoscopic services across the United States.

\section{Introduction}

Since the late 1970 s when the Mayo Clinic first described nurses performing sigmoidoscopy [1], interest in allowing nonphysicians to perform routine gastrointestinal procedures has increased [2,3]. Given the experience with that procedure, several studies have documented the safety and quality of colonoscopies done by non-physicians [4-9]. Based on those data, the British Society of Gastroenterology updated their recommendation in 2005 to endorse non-physician-performed colonoscopy [10]. In the United States, however, the American Society for Gastrointestinal Endoscopy (ASGE) in 2009 issued a practice guideline that states there is insufficient evidence to endorse non-physician-performed colonoscopy or upper endoscopy [11].

To date there are few studies [4] assessing non-physicianperformed upper endoscopy and none that has been performed in the United States. This paucity of data is likely attributable to the fact that so few non-physicians [12] perform upper endoscopy and the lack of standard quality metrics for upper endoscopy. With passage of the Affordable Care Act leading to increasing numbers of patients accessing health care services [13], there will likely be a need for more diagnostic upper endoscopies. Therefore, it is important to assess whether nonphysician endoscopists could be trained to meet this growing need. 
The limited data describing non-physician-performed upper endoscopy come from the United Kingdom and Africa and suggest that upper endoscopy performed by non-physicians is both safe and effective [14-17]. In addition, a recent meta-analysis of non-physician-performed endoscopy suggested that outcomes are similar to procedures performed by physicians [4]. Given the paucity of data for upper endoscopy, we sought to examine the quality data for upper endoscopies performed by nurse practitioners (NPs) at a large safety-net hospital. NPs are advanced practiced nurses who have acquired the expert knowledge base, complex decision-making skills and clinical competencies for expanded practice, the characteristics of which are shaped by the context and/or country in which they are credentialed to practice [18]. The majority of NPs have a master's degree and have trained longer compared with the majority of other non-physician endoscopists who participated in other studies. We hypothesized that upper endoscopies performed by NPs would meet the same standards as physicianperformed upper endoscopy, according to recognized safety and quality standards in the United States [19].

\section{Methods and materials}

\section{Study design}

The study was a retrospective chart review of upper endoscopies performed by 3 NPs at the Zuckerberg San Francisco General Hospital and Trauma Center (ZSFG). Data were abstracted from endoscopy reports, pathology and clinical reports present in the electronic medical record.

\section{Study setting}

ZSFG is a safety-net institution (i. e., provides a significant level of care to low income, uninsured, and vulnerable populations) affiliated with the University of California, San Francisco. Patients are ethnically diverse (20\% African American, $20 \%$ Asian/Pacific Islander, 25\% Caucasian, and 30\% Hispanic), and many are immigrants with more than 20 different languages spoken by patients. Approximately $36 \%$ of outpatients at ZSFG lack insurance, 34\% have MediCal (California's Medicaid program), $16 \%$ have Medicare, and $14 \%$ report commercial payers or other sources. The ZSFG Gastroenterology Division receives 5,300 referrals annually for a wide spectrum of gastrointestinal related conditions and performs over 4,000 endoscopic procedures per year.

\section{Training}

At the Zuckerberg San Francisco General Hospital, a program was developed in 2006 to train a NP to perform colonoscopies and expanded to include upper endoscopy in 2010. After the first NP was trained, 2 additional NPs were trained in both procedures, one in 2011 and the other in 2012. All 3 NPs had master's degrees and had practiced for several years prior to being selected to join the endoscopy unit. One of the NPs had 2 years of experience performing flexible sigmoidoscopy. One NP had been trained on colonoscopy first before learning upper endoscopy while the other 2 NPs learned colonoscopy and upper endoscopy at the same time.
Training for the NPs included a structured curriculum of readings and ASGE endoscopy videos related to the background and practice of colonoscopy and upper endoscopy which occurred over a four-month time period. The curriculum was prepared by the Director of Clinical Gastroenterology in accordance with ASGE guidelines. In addition, the NPs received lectures on topics related to upper endoscopy. Following the curriculum, the NPs underwent hands-on training by performing upper endoscopies under direct observation by a physician endoscopist, all of whom had experience in training gastroenterology fellows in endoscopy. The NPs were proctored for 150 upper endoscopies prior to being assessed for competency in upper endoscopy in accordance with ASGE recommendations [20]. Once they achieved this competence, the NPs were allowed to perform upper endoscopies independently.

During independent practice, the NPs underwent direct observation and auditing of their documentation on a quarterly basis. The NPs only performed endoscopy on outpatients scheduled for a diagnostic procedure for common indications such as dyspepsia, gastroesophageal reflux disease, follow-up of a gastric ulcer, or dysphagia. When a NP performed an endoscopy, there was always an available physician endoscopist to assist with therapeutic maneuvers or to provide a second opinion. If a physician endoscopist was called, the NP would add the physician to the endoscopy report; however, no documentation to explain the reason for physician participation was required. The NPs were allowed to perform diagnostic maneuvers such as mucosal biopsies. All procedures on inpatients or patients requiring therapeutic maneuvers (variceal banding, argon plasma coagulation, endoscopic clips, endoscopic banding, hemostatic injection or sclerotherapy) were performed by a physician endoscopist. In such situations or in the case of an adverse event (AE), a physician endoscopist would assume care of the patient. Following the procedure, the NP documented his or her findings and plan in the endoscopy software (Provation ${ }^{\circledR}$ ), prescribed required medications, followed up pathology results and contacted the patient as appropriate.

\section{Data collection}

Following approval by the UCSF institutional review board, a retrospective chart review of upper endoscopies performed by the 3 NPs from the beginning of October 2010 through the end of February 2013 was performed. Data from all independently performed endoscopies were collected. Endoscopies were excluded if a physician endoscopist was listed as the primary endoscopist. The data were abstracted from the endoscopy report and from the electronic medical record. Data were abstracted for the patient and procedure demographics and documented quality indicators (completed procedure, photo documentation, appropriate biopsies, attending participation, documenting follow up plans, adverse events). The electronic medical record was accessed for pathology data, emergency department visits within 30 days, AEs, and procedure duration. Data collected included date of procedure, name of NP, number of upper endoscopies per NP, indication, duration of procedure (these data were not available for all endoscopies), age of pa- 
tient, race of patient, sex of patient, and American Society of Anesthesiologists (ASA) class, and pathology of biopsies.

\section{Upper endoscopy quality indicators}

Quality indicators were taken from the ASGE and American College of Gastroenterology (ACG) recommendation of practice guidelines [19] and from quality data collected by the endoscopy unit. Many of the indicators endorsed by these societies do not apply to procedures performed by the NPs as they are not credentialed to perform upper endoscopy on inpatients or on patients suspected of having gastrointestinal bleeding. From these practice guidelines the following indicators were selected for the study: obtaining consent, documentation of AEs, documentation of management of antithrombotic therapy, appropriate photo-documentation (gastroesophageal [GE] junction, antrum, retroflexion of the GE junction and the second duodenum), doses of medication, completion of the study, patient instructions, appropriate biopsies of non-bleeding gastric ulcers, measurement of Barrett's esophagus and appropriate biopsy of the esophagus, appropriate biopsy of the duodenum for celiac sprue, frequency of appropriate proton pump inhibitor (PPI) use following diagnosis of gastric or duodenal ulcer. In addition, other quality indicators collected for the study included physician endoscopist participation, gastric biopsies for dyspepsia, esophageal biopsies for dysphagia, duodenal biopsies for iron deficiency anemia, biopsies of any abnormality noted, and appropriate recommendation for PPI.

For organization purposes, quality indicators were divided into 3 categories: 1) pre-procedure quality indicator, which included obtaining consent, documented use of anticoagulant/ antiplatelet prior to procedure; 2) intra-procedure indicators included completion of procedure, photo-documentation, biopsies taken for appropriate indication, and attending assistance, and 3) post-procedure quality indicators included documentation of discharge plan, complication and visits to the emergency department.

\section{Statistical analysis}

Statistical analysis was performed for comparisons among all NPs performing upper endoscopy and an assessment of individual NP performance over time with respect to quality indicators. Proportions and means were used to produce summary statistics. We used the Student $t$ test to compare the means of continuous variables, the chi-square or Fisher's exact test, as appropriate to compare proportions. A $P$ value $<0.05$ was the threshold used for statistical significance. To assess individual NP performance over time, we used individual logistic regression analyses to univariably compare the means of blocks of 20 procedures by each NP. We report odds ratios and $95 \%$ confidence intervals $(\mathrm{Cl})$ for all unvariate and multivariable analyses. A $95 \% \mathrm{Cl}$ not overlapping 1 , and $P$ value $<0.05$, was the threshold for statistical significance. The analyses were performed using Stata (Stata version 11, Statacorp, College Station, TX).

\section{Date reporting}

To protect the identity of our NPs, the order of NPs, as reported in this study, was randomized.

\section{Ethical considerations}

The Committee on Human Research at the University of California San Francisco approved this study.

\section{Results}

A total of 333 upper endoscopies were performed by the 3 NPs (distribution of 166,44 , and 123 , respectively) during the study period. Characteristics of the endoscopies by provider are outlined in $>$ Table 1. The most common indication was dyspepsia, reflux or nausea (55.0\%) followed by iron deficiency anemia (20.1\%). Most patients were Asian (46.0\%), had a mean age of 52.8 years, and had a mean ASA score of 1.8. Mean procedure duration was $6.4 \pm 0.2$ minutes with mean doses of fentanyl $80.7 \mathrm{mcg}( \pm 27.7 \mathrm{mcg})$ and midazolam $3.2 \mathrm{mg}( \pm 1.1 \mathrm{mg})$ administered for sedation.

\section{Pre-procedure quality indicators}

- Table 2 highlights results of the quality indicators for NPs performing upper endoscopy. For the quality measures prior to the start of endoscopy, consent was performed in $100 \%$ of cases and the patient was asked about prior use of anticoagulant or antiplatelet agents in $99.4 \%$ with no significant differences among the 3 NPs.

\section{Intra-procedure quality indicators}

Of the 333 upper endoscopies, $98.2 \%$ were completed to the second portion of the duodenum. Five of the 6 cases that were not completed were aborted due to patient intolerance and 1 was aborted due to an obstructing adenocarcinoma. In most cases, photo-documentation of required anatomical landmarks was performed: GE junction in forward view (85.8\%), GE junction in retroflexed view (84.2\%), antrum (82.1\%) and duodenum (80.9\%). NP2 took consistently fewer photos compared to NP1 and NP3. In logistic regression analysis, the odds of appropriate photo-documentation increased with advancing procedural experience. Odds ratios (by blocks of 20 procedures) were as follows: GE junction in forward view: $1.56(95 \% \mathrm{Cl}$ $1.29,1.91)$; GE junction in retroflexed view: 1.46 (95\% Cl 0.23 , 1.75), antrum: 1.49 (95\% Cl 1.26, 1.77); duodenum: 1.35 (95\% $\mathrm{Cl} 1.16,1.57)$. In regards to appropriate biopsies per indication, the NPs performed the indicated biopsies in more than $90 \%$ of procedures: $90.9 \%$ gastric ulcers were biopsied, $94.3 \%$ of patients with dyspepsia were biopsied for Helicobacter pylori, $95.5 \%$ of patients with iron deficiency had duodenal biopsies performed for celiac disease, and $83.3 \%$ of patients with dysphagia underwent esophageal biopsy. A physician endoscopist was required during the procedure $22.5 \%$ of the time, more often for NP3. Overall, participation by a physician endoscopist did not decrease significantly with increasing experience.

\section{Post-procedure quality indicators}

Following the procedure, a follow-up discharge plan was noted $99.4 \%$ of the time. There was 1 immediate complication and 1 complication within 30 days. The immediate complication was a post-procedural bleed of a newly diagnosed gastrointestinal 
- Table 1 Characteristics of upper endoscopies by provider.

\begin{tabular}{|c|c|c|c|c|}
\hline & NP1 & NP2 & NP3 & All NPs \\
\hline Total procedures, $\mathrm{N}$ & 166 & 44 & 123 & 333 \\
\hline \multicolumn{5}{|l|}{ Indication, No.(\%) } \\
\hline - Dyspepsia, reflux, or nausea & $81(48.8)$ & $26(59.1)$ & $76(61.8)$ & $183(55.0)$ \\
\hline - Iron deficiency anemia & $37(22.3)$ & $7(15.9)$ & $23(18.7)$ & $67(20.1)$ \\
\hline - Dysphagia & $4(2.4)$ & $2(4.6)$ & $3(2.4)$ & $9(2.7)$ \\
\hline - Ulcer follow-up & $8(4.8)$ & $2(4.6)$ & $7(5.7)$ & $17(5.1)$ \\
\hline - Weight loss & $13(7.8)$ & $2(4.6)$ & $4(3.3)$ & $19(5.7)$ \\
\hline - Barrett's esophagus & $2(1.2)$ & $1(2.3)$ & $2(1.6)$ & $5(1.5)$ \\
\hline - Other & $19(11.5)$ & $4(9.1)$ & $8(6.5)$ & $31(9.3)$ \\
\hline \multicolumn{5}{|l|}{ Patient race/ethnicity, No. (\%) } \\
\hline - White & $23(13.9)$ & $5(11.4)$ & $20(16.3)$ & $48(14.4)$ \\
\hline - Black & $17(10.2)$ & $4(9.1)$ & $8(6.5)$ & $29(8.7)$ \\
\hline " Hispanic & $60(36.1)$ & $8(18.2)$ & $38(30.9)$ & $106(31.8)$ \\
\hline - Asian & $63(38.0)$ & $23(52.3)$ & $52(42.3)$ & $138(41.4)$ \\
\hline - Other & $3(1.8)$ & $4(9.1)$ & $5(4.1)$ & $12(3.6)$ \\
\hline Age, mean (SD) & $52.6(11.8)$ & $55.9(9.4)$ & $52.0(12.8)$ & $52.8(11.9)$ \\
\hline \multicolumn{5}{|l|}{ Sedation used, mean (SD) } \\
\hline - Fentanyl (mcg) & $85.4(31.8)$ & $72.2(18.5)$ & $76.4(21.9)$ & $80.7(27.7)$ \\
\hline . Midazolam (mg) & $3.4(1.3)$ & $2.8(0.7)$ & $3.0(0.9)$ & $3.2(1.1)$ \\
\hline ASA class, mean (SD) & $1.7(0.56)$ & $1.8(0.4)$ & $2.0(0.2)$ & $1.8(0.45)$ \\
\hline - Length of case in minutes, mean (SD) & $5.9(2.5)$ & Not available & $7.5(3.2)$ & $6.4(2.9)$ \\
\hline
\end{tabular}

stromal tumor. A physician endoscopist had assisted on the case and with the biopsies. Eleven patients (3.3\%) visited the emergency department within 30 days of the endoscopy but none, other than the above complication, was for a cause related to the procedure.

\section{Discussion}

Our study, which is the first to investigate NPs performing upper endoscopy in the United States, suggests that NPs are able to perform upper endoscopy safely and according to national quality guidelines. A number of important conclusions can be drawn from our study. First, by adhering to a systematic training program, NPs can be sufficiently trained to perform upper endoscopies in the outpatient setting. Secondly, NPs can safely perform high-quality upper endoscopies in an independent fashion under the guidance of an attending physician. These findings support incorporation of non-physicians alongside physicians to help meet the growing demand for endoscopic services across the United States.

One important finding from our study is that not only can non-physicians safely perform upper endoscopies, but that they can also perform a high-quality procedure as well. Previous research has shown the feasibility and safety of non-physicians performing upper endoscopy [4,14-16]; whereas our study has expanded this research by investigating the quality of non-physician-performed upper endoscopy. Our study highlights that all NPs were able to successfully satisfy multiple quality indicators in the pre-procedure, intra-procedure and post-procedure process. Of note, there was some variation noted among the 3 NPs in terms of photo-documentation and performing biopsies for several indications. It is important to point out that all 3 NPs showed improvement over time with respect to these quality indicators. A possible explanation for these differences is that the NPs were trained prior to the introduction of the ASGE/ACG guidelines and they may not have been familiar with what needed to be documented. After the guidelines were published, the NPs received additional training on these approved quality guidelines. Moreover, while the NPs met quality indicators for upper endoscopies with few AEs, it is important to note that their procedure duration was not excessive. The mean NP procedure duration was 6.2 minutes which compares with other reported times by physicians performing upper endoscopy $[15,17]$ and that time decreased with in- 
- Table 2 Procedure quality data.

\begin{tabular}{|c|c|c|c|c|c|}
\hline & NP1 & NP2 & NP3 & All NP's & $P$ value \\
\hline \multicolumn{6}{|l|}{ Pre-procedure quality indicator } \\
\hline Consent obtained, No. (\%) & $166(100.0)$ & $44(100.0)$ & $123(100.0)$ & $333(100.0)$ & - \\
\hline $\begin{array}{l}\text { Use of anticoagulant or antiplatelet agent } \\
\text { documented, No. (\%) }\end{array}$ & $166(100.0)$ & $43(97.7)$ & $122(99.2)$ & $331(99.4)$ & 0.21 \\
\hline \multicolumn{6}{|l|}{ Intra-procedure quality indicator } \\
\hline Complete to second duodenum, No. (\%) & $160(96.4)$ & $44(100.0)$ & $123(100.0)$ & $327(98.2)$ & $\begin{array}{l}0.05 \text { overall } \\
0.03 \text { NP3-NP1 }\end{array}$ \\
\hline \multicolumn{6}{|l|}{ Photo- documentation, No. (\%) } \\
\hline - GEjunction & $157(95.2)$ & $9(20.5)$ & $119(96.8)$ & $285(85.8)$ & $\begin{array}{l}<0.001 \text { overall } \\
<0.001 \mathrm{NP} 1-\mathrm{NP2} \\
<0.001 \mathrm{NP2}-\mathrm{NP3}\end{array}$ \\
\hline - Retroflexion & $153(93.9)$ & $9(20.5)$ & $116(94.3)$ & $278(84.2)$ & $\begin{array}{l}<0.001 \text { overall } \\
<0.001 \text { NP1-NP2 } \\
<0.001 \text { NP2-NP3 }\end{array}$ \\
\hline - Antrum & $149(91.4)$ & $6(13.6)$ & $116(94.3)$ & $271(82.1)$ & $\begin{array}{l}<0.001 \text { overall } \\
<0.001 \text { NP1-NP2 } \\
<0.001 \text { NP2-NP3 }\end{array}$ \\
\hline - Duodenum & $149(92.0)$ & $5(11.4)$ & $112(91.1)$ & $266(80.9)$ & $\begin{array}{l}<0.001 \text { overall } \\
<0.001 \text { NP1-NP2 } \\
<0.001 \text { NP2-NP3 }\end{array}$ \\
\hline \multicolumn{6}{|l|}{ Biopsy taken, No. (\%) } \\
\hline - Gastric ulcer & $0 / 1(0.0)$ & $2 / 2(100.0)$ & $8 / 8(100.0)$ & $10 / 11(90.9)$ & $\begin{array}{l}0.004 \text { overall } \\
0.003 \text { NP1-NP3 }\end{array}$ \\
\hline - Dyspepsia & $70 / 73(95.9)$ & $13 / 18(72.2)$ & $67 / 68(98.5)$ & $150 / 159(94.3)$ & $\begin{array}{l}<0.001 \text { overall } \\
0.001 \mathrm{NP} 1-\mathrm{NP} 1 \\
<0.001 \mathrm{NP2}-\mathrm{NP3}\end{array}$ \\
\hline - Dysphagia & $2 / 4(50.0)$ & $2 / 2(100.0)$ & $6 / 6(100.0)$ & $10 / 12(83.3)$ & 0.09 \\
\hline - Iron deficiency anemia & $37 / 37(100.0)$ & $5 / 7(71.4)$ & $21 / 22(95.5)$ & $63 / 66(95.5)$ & $\begin{array}{l}0.004 \text { overall } \\
0.001 \text { NP1-NP2 }\end{array}$ \\
\hline $\begin{array}{l}\text { Physician endoscopist assistance required, } \\
\text { No. (\%) }\end{array}$ & $19(11.5)$ & $6(13.6)$ & $50(40.7)$ & $75(22.5)$ & $\begin{array}{l}<0.001 \text { overall } \\
<0.001 \text { NP2-NP3 } \\
0.001 \text { NP1-NP3 }\end{array}$ \\
\hline \multicolumn{6}{|l|}{ Post-procedure quality indicator } \\
\hline Immediate complication, №. (\%) & $1(0.6)$ & $0(0.0)$ & $0(0.0)$ & $1(0.3)$ & 0.60 \\
\hline Discharge plan documented, No. (\%) & $165(99.4)$ & $43(97.7)$ & $123(100.0)$ & $331(99.4)$ & 0.25 \\
\hline Complication within 30 days, No. (\%) & $1(0.6)$ & $0(0.0)$ & $0(0.0)$ & $1(0.3)$ & 0.60 \\
\hline Patient went to ED within 30 days, No. (\%) & $7(4.2)$ & $0(0.0)$ & $4(3.3)$ & $11(3.3)$ & 0.38 \\
\hline
\end{tabular}

creasing experience of the NPs. Thus, our study illustrates that non-physicians can safely perform upper endoscopy, successfully meet all accepted quality indicators for upper endoscopy and do so at no significant increase in procedure time.

One critical question that arises from the study is the need for physician endoscopist involvement, both in terms of time required by the attending and cost of this resource. From our study, just under one-quarter of upper endoscopies involved an attending physician in procedures performed by an NP. In addition, there was tremendous practice variation with attend- ing involvement with a high rate of attending assistance for 1 NP compared to the other NPs. As this was a retrospective study, physician participation was recorded but the reasoning for the participation was not. The NPs are required to have the assistance of a physician for certain therapeutic maneuvers, and therefore, physician participation is not necessarily an indication that the NP only requested a second opinion. Also, the amount of time that the physician spent in each case was not recorded and likely varied. 
Furthermore, the financial impact of the supervising physician of the NPs is an important consideration. For example, there have been several conflicting studies regarding the costeffectiveness of all endoscopies performed by non-physicians $[8,21,22]$. Yet none of these studies has incorporated the need for physician assistance. In addition, none of these studies have addressed the differences in the cost and time to train physicians compared to non-physicians which are substantially different. The rate of physician participation and the difference in the cost of training would likely have a significant impact on the overall cost of implementing such a program. Therefore, future studies investigating the cost-effectiveness of non-physician endoscopy should attempt to capture these differences in order to make a better estimate.

One remaining issue in terms of physicians and NPs performing endoscopy may relate to differences in education of physicians compared with non-physicians. This was recently addressed by Stephens et al. [22] in a systematic review whereby they argued that the cognitive competency of physicians and nonphysicians is significantly different due to different types of training. In this review, it was raised that physicians tend to put the patient in the context of the overall clinical picture while nurses tend to work along protocols. Because cognitive competence requires more comprehensive training, which is typically reserved for physicians, one could argue that non-physician endoscopists may not be as effective in delivering care. In the delivery of care, providers must be able to do more than perform a procedure as they as need to evaluate the pathology, consider the patient's clinical risks, and recommend further treatment. Put another way, endoscopists need to be clinicians, not simply technicians. Stephens' conclusion was that physicians are better trained to handle these complex situations compared to non-physicians, who can rely on protocols that can only be applied to for simple tasks. Such a difference in cognitive competence possibly led to the finding in the MINuET Trial in the U.K. that showed a small gain in quality adjusted life years (QALYs) in the physician group compared to the nurse endoscopists [21]. In that article, the authors argued that the difference in QALYs was likely due to an increased number of follow-up tests ordered on patients who saw a non-physician for their procedure. The authors did note that as the differences in health outcomes were statistically similar, there was some uncertainty to the economic analysis. Their conclusion was that while physicians were currently more cost-effective, it would be necessary to reevaluate the cost-effectiveness over time as non-physician endoscopists gained more experience with performing procedures and ordering appropriate follow up tests for their patients.

Although the concern regarding cognitive competence is not without merit, we would argue that cognitive competence, like procedural competence, can be taught over time. Non-physicians have been performing flexible sigmoidoscopies for decades, but they are relatively new to performing upper endoscopies. As shown in our study, well-trained non-physicians can independently perform the procedure safely and according to quality standards. Non-physicians practice under the supervision of physicians and should be trained in cognitive aspects as well as the procedural to deliver the highest quality of care possible. At our center, the NPs all had master's degrees and had practiced in the challenging environments of the emergency medicine department and the intensive care unit (ICU) prior to their training in endoscopy. Their training was similar to those of the gastroenterology fellowship trainees except that the NP training focused solely on outpatients. As there is little published on the training protocols of non-physician endoscopists internationally, it is unclear how training of our NPs differed. However, in other medical fields nurse anesthetists and nonphysicians in the ICU have been trained to manage medically complex patients and have become an accepted part of medical teams in these specialties [23-25]. As these non-physicians have been train to deal with cognitively challenging patients, we feel that there is a role for non-physicians in endoscopy.

One possible solution, as suggested in a recent review [26], is to test incorporation of non-physicians in a smaller setting before incorporating on a larger scale. The authors acknowledge the competing concerns that the number of procedures are increasing but it is not known whether non-physicians can consistently deliver high-quality care or if their participation is cost-effective. Our study is unable to answer all these concerns but shows that a group of highly trained NPs are able to function independently within a defined scope of work and under the indirect supervision of physicians. Future studies of the cost-effectiveness of this approach and assessing the cognitive competency of the non-physicians would be useful to see whether this model can be expanded.

Our study has several limitations. Because it only examined NP procedures and at a single center, there was no comparison group of physician-performed endoscopies and the findings may not be generalizable. As well, there was no way to confirm the findings of the NPs because there was not a large enough group of patients who underwent a repeat procedure by a physician nor do we record endoscopies for review. However, the NPs have quarterly audits by a physician endoscopist to ensure quality endoscopy. In addition, we did not examine satisfaction of or acceptance by patients of a non-physician performing their procedure. Although previous work has shown high patient satisfaction with NPs performing colonoscopy [7], it would be important to note this information for the population at a safety-net hospital. Another limitation is that there is no gold-standard for quality metrics for upper endoscopy. The ASGE/ACG guidelines pertain mostly to therapeutic procedures that are not performed by our NPs. Nonetheless, the quality metrics selected for this study seem to be generalizable and accepted measures of quality.

\section{Conclusion}

In summary, our study is the first from the United States to show that trained NPs were able to perform high-quality and safe upper endoscopies. Our data support continued discussion on incorporating and augmenting gastroenterology practices with expanding the role of non-physicians to assist with the high volume of endoscopic procedures. Incorporation of NPs into the endoscopy schedule may possibly lead to increased 
procedure volume and access and allow gastroenterologists to focus their attention on more complex and demanding procedures/cases. Importantly, continued research is required to determine optimal teaching methods and patient acceptance of this practice and to begin the development of standard guidelines for the scope of practice for non-physicians performing endoscopy. Future studies should compare NPs to physicians and assess costs associated with both groups. In this study, the first from the United States, trained NPs were able to safely perform upper endoscopies and meet general quality standards.

\section{Competing interests}

None

\section{References}

[1] Spencer RJ, Ready RL. Utilization of nurse endoscopists for sigmoidoscopic examinations. Dis Colon Rectum 1977; 20: 94 - 96

[2] Hutfless S, Kalloo AN. Screening Colonoscopy: A New Frontier for Nurse Practitioners. Clin Gastroenterol Hepatol 2013; 11: 106-108

[3] Norton C, Grieve A, Vance M. Nurse delivered endoscopy. BM] 2009; 338: a3049

[4] Day LW, Siao D, Inadomi JM et al. Non-physician performance of lower and upper endoscopy: a systematic review and meta-analysis. Endoscopy 2014; 46: $401-410$

[5] Hui A], Lau JY, Lam PP et al. Comparison of colonoscopic performance between medical and nurse endoscopists: a non-inferiority randomised controlled study in Asia. Gut 2015; 64: 1058 - 1062

[6] Koornstra J], Corporaal S, Giezen-Beintema WM et al. Colonoscopy training for nurse endoscopists: a feasibility study. Gastrointest Endosc 2009; 69: 688-695

[7] Limoges-Gonzalez M, Mann NS, Al-Juburi A et al. Comparisons of screening colonoscopy performed by a nurse practitioner and gastroenterologists: a single-center randomized controlled trial. Gastroenterol Nurs Off J Soc Gastroenterol Nurses Assoc 2011; 34: 210 - 216

[8] MassI R, van Putten PG, Steyerberg EW et al. Comparing quality, safety, and costs of colonoscopies performed by nurse vs physician trainees. Clin Gastroenterol Hepatol Off Clin Pract J Am Gastroenterol Assoc 2014; 12: $470-477$

[9] van Putten PG, Ter Borg F, Adang RP et al. Nurse endoscopists perform colonoscopies according to the international standard and with high patient satisfaction. Endoscopy 2012; 44: 1127-1132

[10] Swarbrick E, Harnden S, Hodson R et al. A Report of the Working Party of the British Society of Gastroenterology (Non-medical Endos- copists). Loughborough, Leicestershire; 2005: Available from: http:// www.bsg.org.uk/images/stories/docs/clinical/guidelines/endoscopy/ endo_\%20nonmed.pdf

[11] Ikenberry SO, Anderson MA, Banerjee $S$ et al. Endoscopy by nonphysicians. Gastrointest Endosc 2009; 69: 767-770

[12] Sharma VK, Coppola AG Jr, Raufman JP. A survey of credentialing practices of gastrointestinal endoscopy centers in the United States. J Clin Gastroenterol 2005; 39: 501 - 507

[13] U.S. Department of Health and Human Services, Health Resources and Services Administration, National Center for Health Workforce Analysis. Projecting the Supply and Demand for Primary Care Practitioners Through 2020. Rockville, Maryland: U.S. Department of Health and Human Services; 2013

[14] Smale S, Bjarnason I, Forgacs I et al. Upper gastrointestinal endoscopy performed by nurses: scope for the future? Gut 2003; 52: 1090 - 1094

[15] Williams J, Russell I, Durai D et al. Effectiveness of nurse delivered endoscopy: findings from randomised multi-institution nurse endoscopy trial (MINuET). BMJ 2009; 338: b231

[16] Wilhelm TJ, Mothes H, Chiwewe D et al. Gastrointestinal endoscopy in a low budget context: delegating EGD to non-physician clinicians in Malawi can be feasible and safe. Endoscopy 2012; 44: 174-176

[17] Meaden C, Joshi M, Hollis S et al. A randomized controlled trial comparing the accuracy of general diagnostic upper gastrointestinal endoscopy performed by nurse or medical endoscopists. Endoscopy 2006; 38: $553-560$

[18] ICN Nurse Practitioner / Advanced Practice Nursing Network. Available from: NP and AP Roles.http://international.aanp.org/Practice/ APNRoles

[19] Park WG, Shaheen NJ, Cohen J et al. Quality indicators for EGD. Am J Gastroenterol 2015; 110: 60-71

[20] Faulx AL, Lightdale JR, Acosta RD et al. Guidelines for privileging, credentialing, and proctoring to perform Gl endoscopy. Gastrointest Endosc 2017; 85: 273-281

[21] Williams ], Russell I, Durai D et al. What are the clinical outcome and cost-effectiveness of endoscopy undertaken by nurses when compared with doctors? A Multi-Institution Nurse Endoscopy Trial (MINuET) Health Technol Assess Winch Engl 2006: iii-iv, ix-x, 10: 1- 195

[22] Stephens M, Hourigan LF, Appleyard M et al. Non-physician endoscopists: A systematic review. World J Gastroenterol WJG 2015; 21: $5056-5071$

[23] Matsusaki T, Sakai T. The role of Certified Registered Nurse Anesthetists in the United States. J Anesth 2011; 25: 734-740

[24] Gershengorn HB, Wunsch H, Wahab R et al. Impact of Nonphysician Staffing on Outcomes in a Medical ICU. Chest 2011; 139: 1347-1353

[25] Kleinpell RM. Acute care nurse practitioner practice: results of a 5-year longitudinal study. Am J Crit Care 2005; 14: 211 - 219

[26] Pfeifer UG, Schilling D. Non-physician endoscopy: How far can we go? Visc Med 2016; 32: 0113-20 